\title{
Cognitive and neural determinants of response strategy in the dual-solution plus-maze task
}

\author{
Elvira De Leonibus, ${ }^{1,2,3,4,7,8}$ Vivian J. A. Costantini, ${ }^{1,2,7}$ Antonio Massaro, ${ }^{1,2}$ \\ Georgia Mandolesi, ${ }^{5}$ Valentina Vanni, ${ }^{5}$ Siro Luvisetto, ${ }^{6}$ Flaminia Pavone, ${ }^{6}$ \\ Alberto Oliverio, ${ }^{1,2,6}$ and Andrea Mele $\mathrm{e}^{1,2,6}$
}

\begin{abstract}
${ }^{1}$ Dipartimento di Biologia e Biotecnologie, Università degli Studi di Roma "La Sapienza," 00185 Rome, Italy; ${ }^{2}$ Centro di Ricerca in Neurobiologia-D. Bovet, Università degli Studi di Roma "La Sapienza," 00185 Rome, Italy; ${ }^{3}$ Institute of Genetics and Biophysics (IGB), CNR, 80131 Naples, Italy; ${ }^{4}$ Telethon Institute of Genetics and Medicine (TIGEM), 80131 Naples, Italy; ${ }^{5}$ Laboratory of Neuroplasticity, CERC-Fondazione Santa Lucia, 00143 Rome, Italy; ${ }^{6}$ Istituto di Neuroscienze, CNR-CERC, 00143 Rome, Italy
\end{abstract}

\begin{abstract}
Response strategy in the dual-solution plus maze is regarded as a form of stimulus-response learning. In this study, by using an outcome devaluation procedure, we show that it can be based on both action-outcome and stimulus-response habit learning, depending on the amount of training that the animals receive. Furthermore, we show that deactivation of the dorsomedial and the dorso-lateral striatum with Botulinum neurotoxin $A$, mimicked or abolished, respectively, the effects of practice on the sensitivity of the response strategy to outcome devaluation. These findings have relevant implications for the understanding of the learning mechanisms underlying different overt behaviors in this widely used maze task.
\end{abstract}

[Supplemental material is available for this article.]

The dual-solution plus-maze task developed by Tolman et al. (1946) has been used as a valuable tool to distinguish between goal-directed actions and habitual responses. In this task, rodents are trained to retrieve food from a consistently baited arm, starting always from the same start box. Using this procedure, they may learn to find food in a particular place in space (place strategy) or to make a particular body turn (response strategy). However, by testing them from the start box used during training does not allow a discrimination of the kind of strategy used by the animals. But the strategy they use can be resolved by submitting them to a probe test from the opposite arm. It has been demonstrated that with increasing training, normal rats shift from a place to a response strategy (RS) (Packard and McGaugh 1996; Packard 1999; Yin and Knowlton 2004). There is a wealth of studies in the literature, both in rats and mice, which adopts the RS to study the neurobiology of stimulus-response (S-R) habit learning (Packard 1999; Passino et al. 2002; Colombo et al. 2003; Compton 2004; Middei et al. 2004a, b; Brightwell et al. 2008; Kheirbek et al. 2009; Moustgaard and Hau 2009; Ferragud et al. 2010). Nevertheless, the kind of learning mechanism underlying RS has never been studied before. Recent experimental evidence in instrumental tasks showed that, independently of the behavior observed, the same response can rely on either action-outcome (A-O) or S-R association, respectively, controlled by reward expectancy or by antecedent stimuli. Thus, a stringent criterion to discriminate the learning mechanism underlying the RS would be to test its sensitivity to reward devaluation (Vanderschuren and Everitt 2004; Yin et al. 2004, 2005a,b; 2006).

\footnotetext{
${ }^{7}$ These two authors contributed equally to this work.

${ }^{8}$ Corresponding author.

E-mail deleonibus@igb.cnr.it; fax 39-081-6132706.

Article is online at http://www.learnmem.org/cgi/doi/10.1101//m.2074311.
}

Toward this result, in this study food-restricted $(80 \%-85 \%$ of their free-feeding weight) CD1 adult male mice (Charles River, Italy) were trained ( 15 consecutive trials $\times$ day, ITI $=40 \mathrm{sec}$ ) for $26 \mathrm{~d}$ to find a reward (chocolate puffed rice) in a constantly baited arm (e.g., west), always starting from the same start box (south) (Fig. 1A, Training panel). When animals entered the unbaited arm they were confined there for $15 \mathrm{sec}$ (with no reward available) and the response was scored as incorrect trials. Entry into the baited arm was rewarded with one grain of chocolate and scored as a correct trial (Fig. 1A, Training panel). On day 27, the mice were tested for the acquired strategy, releasing them from a novel arm (north) (Fig. 1A, Probe 1 panel). Animals entering the arm where the reward was located during training were designed as "place;" on the contrary, animals making the same turning response as during training were designed as "response" or using response strategy (RS) (Fig. 1A, Probe 1 panel).

As shown in Figure 1B, 82\% (22 out of 26 animals, $P=0.001$ ) of the animals approached the goal box opposite to the one consistently baited during training. This result confirms that longlasting training favors the use of RS (a specific body turn) as compared with a place strategy (Packard 1999; Yin and Knowlton 2004).

To test the sensitivity of the RS to reward value change, mice displaying RS on day 27 were submitted for five consecutive days to a reinforcement devaluation procedure by pairing the chocolate reward with Lithium Chloride ( $\mathrm{LiCl}$ ) once the animals were placed in a waiting cage after training (Supplemental material). Control mice (valued) were administered with saline (Fig. 1A, Devaluation panel). This procedure, while inducing taste aversion to the reward, as shown by the significant reduction in chocolate consumption observed both in the waiting cage and in the maze (Supplemental Fig. S1A,B), did not affect the number of correct trials from the training arm (Fig. 1C). In fact, the percentage of correct trials expressed by reward-devalued mice on day 5 of reward 

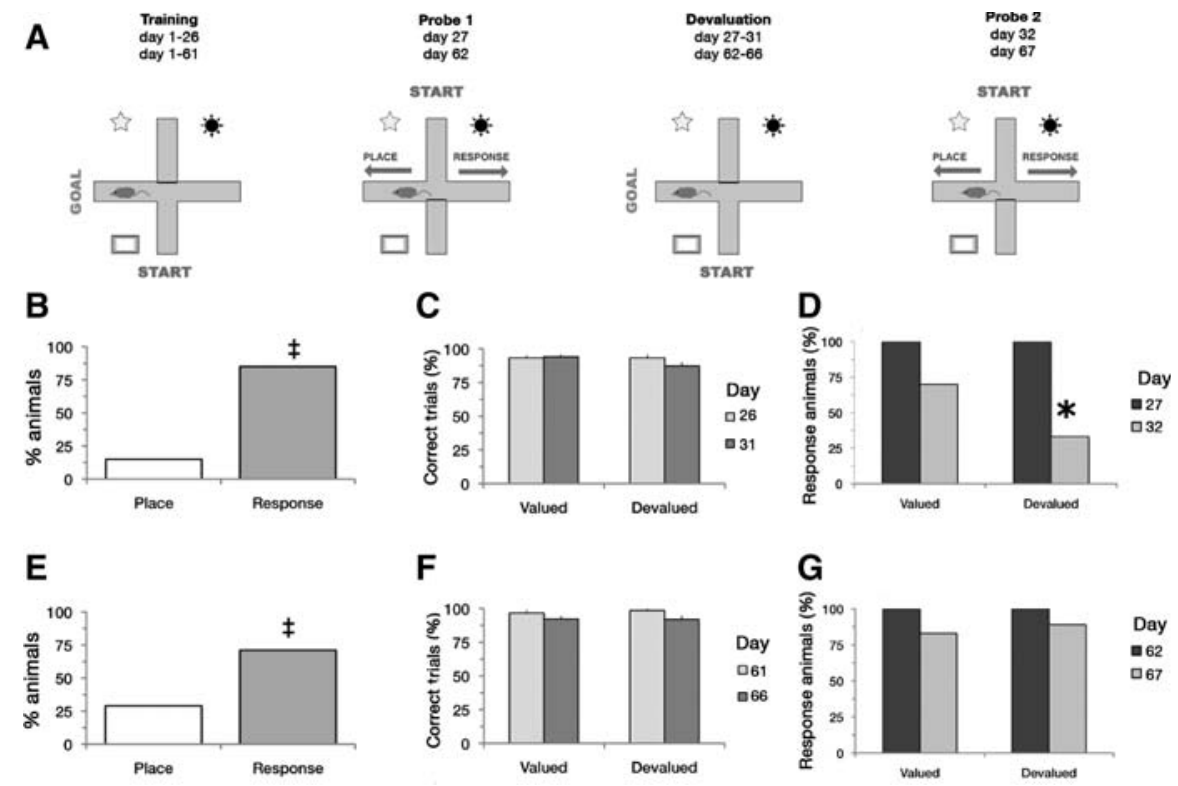

Figure 1. (A) Schematic representation of the apparatus, the training, and the testing procedures with the indication of the start and the goal arm in different stages. The apparatus was a plus maze, consisting of four radial arms (north, south, east, west) with clear Plexiglas walls and a black-opaque Plexiglas floor. The maze was placed in the center of a black square-shaped gazebo measuring $2 \times 2 \mathrm{~m}$ and enlightened by a lamp $(60 \mathrm{~W})$ positioned directly above the maze. (B) Percentage of mice emitting a place or a response on probe test 1 (day 27) in the dual-solution plus-maze task, showing a preferential use of the response strategy. (C) Percentage of correct trials on day 26 (before devaluation) and on day 31 (on day 5 of devaluation) in the valued and devalued groups. (D) Percentage of mice using a response strategy on probe test 1 (day 27), maintaining their response on probe test 2 (day 32 ) in the valued and devalued group. Devalued, but not valued mice shifted their response. (E) Percentage of mice using a place or a response strategy after overtraining on probe test 1 (day 61). After $61 \mathrm{~d}$ of training most animals use a RS. ( $F)$ In overtrained mice the percentage of correct trials on the last devaluation day (day 66) did not differ from percentage of correct trials emitted on last training day (day 61). (G) When response learners mice trained for $61 \mathrm{~d}$ were submitted to outcome devaluation, no change in turning response was observed in the second probe test (on day 67). ( $\$$ ) $P<0.05$ Place vs. Response on probe test 1 (day 27 or 62 ); ( $\left.{ }^{*}\right) P<0.05$ before and after devaluation-day 27 vs. day 32 .

devaluation (day 31) did not differ from that of the same group before devaluation or that of the valued controls (outcome $F_{(1,20)}=1.824 ; \quad P=$ n.s. $], \quad$ days $\quad\left[F_{(1,20)}=1.924 ; \quad P=\right.$ n.s. $]$, outcome $\times$ days $\left[F_{(1,20)}=2.982 ; P=\right.$ n.s. $]$ ) (Fig. 1 C). This result demonstrates that the turning response emitted by the animals, starting from the familiar start box, is insensitive to reward devaluation, consistent with an S-R habit learning. The same mice were then submitted to an additional probe test (day 32), in which they were released from the start box opposite to the one used during training (Fig. 1A, Probe 2 panel). Most mice in the control group maintained their turning response (Fig. 1D). The behavior of this group confirmed that RS is insensitive to maze arm change, and, therefore, is not guided by spatial information. Unexpectedly, the devalued group, when released from the novel arm, shifted behavior, turning in the opposite direction (Fig. 1D), $\left(\chi^{2}\right.$ for the devalued and valued groups being, respectively: $\chi^{2}=$ 5.33, $P=0.020$, and $\chi^{2}=0.90, P=$ n.s.).

These data show that the RS in the plus maze is insensitive to changes in the reward value in animals released from the training arm, but sensitive to changes from the novel arm. This means that the turning behavior in the two testing conditions has a different sensitivity to reward devaluation and, therefore, might be based on distinct learning mechanisms. Since the north start arm could be distinguished from the south arm only if the animal codified the spatial relationship between all of the other available stimuli in the environment ("context related information"), these data suggest that learned responses (from the training arm), insensitive

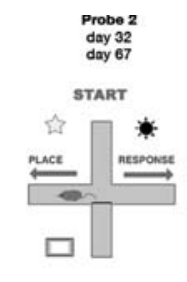

to the reward value change during the initial test conditions, became sensitive when a spatial change occurred. Recent findings demonstrated that when hungry rats are trained on single response, test performance is insensitive to devaluation of the contingent outcome. On the contrary, training on a schedule that offers a choice between responses that yield different outcomes prevents the onset of behavioral autonomy (Kosaki and Dickinson 2010). According to these data, RS animals were not sensitive to reward devaluation from the familiar arm because they were not trained for an alternative; on the contrary, when they were released from the opposite arm, they were challenged by the spatial change and able to use a goal-directed behavior against the acquired habit. These findings prompt the suggestion that when changes in the subjects' motivation failed to modify habits, a simultaneous spatial change might favor the desired behavioral modification.

To test whether further practice could yield a complete behavioral autonomy, in the next experiment we overtrained the mice for $61 \mathrm{~d}$ (915 trials). As expected, most mice $(71 \%, 15$ out of 21 , $P=0.03)$ when challenged from the novel arm on day 62 (probe 1), made the same body turn utilized during training (RS) (Fig. 1E). Mice utilizing the RS were then submitted to the reward devaluation procedure for $5 \mathrm{~d}$, as previously described. After devaluation, mice showed reduced food consumption (Supplemental Fig. S1C,D). Despite the acquired taste aversion, the response of the devalued group did not differ from that of the valued controls (Fig. 1F) (Days $\left[F_{(1,20)}=6.72 ; P=0.02\right]$; no significant differences with post-hoc analysis). However, in this study unlike previously, when tested on day 67 (Probe 2) from the novel arm devalued mice showed a response similar to that of the controls (Fig. 1G) Devalued $\left[\chi^{2}=\right.$ $0.11, P=$ n.s. $]$ and valued group $\left[\chi^{2}=0.17, P=\right.$ n.s. $]$ ).

These results demonstrate that overtraining makes RS insensitive to both arm-entering and reward-value changes. Furthermore, the difference observed in the sensitivity to reward value changes from the novel arm in the animals trained $26 \mathrm{vs}$. $61 \mathrm{~d}$ provides the first experimental evidence in the literature that RS acquired after extensive training in the plus maze can be either dependent or independent of the value of the reinforcement. This allows for the conclusion that RS can be based on A-O or S-R (Adams and Dickinson 1981; Adams 1982; Balleine 1992) depending on the amount of training the animals receive. A direct implication of this result is that, although the probe test is generally used to infer the kind of learning mechanism used by the animals relying on RS (Packard 1999; Passino et al. 2002; Colombo et al. 2003; Compton 2004; Middei et al. 2004a, b; Brightwell et al. 2008; Kheirbek et al. 2009; Moustgaard and Hau 2009; Ferragud et al. 2010), the turning behavior from the novel arm is not sufficient to define it by itself.

Recent experimental evidence shows that manipulations of the dorso-medial striatum (DMS) impair A-O learning (Yin and Knowlton 2004; De Leonibus et al. 2005; Yin et al. 2005a), while 


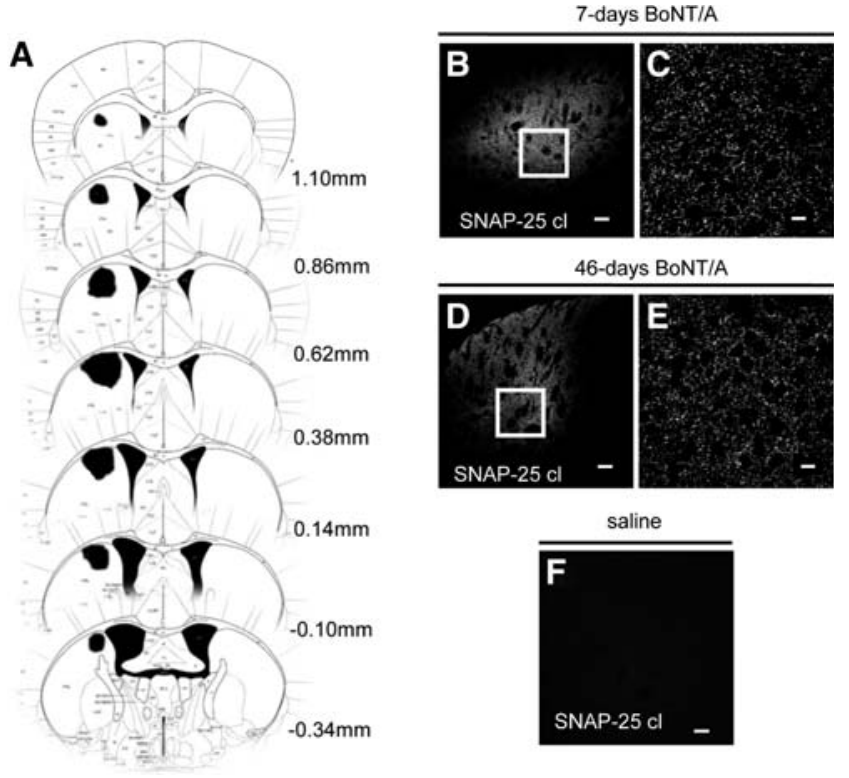

Figure 2. BoNT/A injection in the dorsal striatum. (A) Reconstruction of coronal sections through the dorsal striatum. Numbers represent the anterior-posterior coordinates relative to the bregma according to the mouse brain atlas (Franklin and Paxinos 1997). (B-E) The robust immunostaining for cleaved SNAP-25 (red labeling) shows the effectiveness of BoNT/A treatment at $7 \mathrm{~d}(B, C)$ and $46 \mathrm{~d}$ after the injection $(D, E)$. $C$ and $E$ are a high magnification of the white square in $B$ and $D$, respectively. $(F)$ Staining for cleaved SNAP-25 in control mice injected with saline solution. Scale bar, $B, D$, and $F, 100 \mu \mathrm{m} ; C$ and $E, 10 \mu \mathrm{m}$.

lesions of the dorso-lateral (DLS) component of the striatum induce deficits in S-R habit learning (Yin and Knowlton 2004; Yin et al. 2005b). Therefore, we tested the involvement of the DMS and DLS in mediating RS sensitivity to outcome value change after 26 and $61 \mathrm{~d}$ of training, respectively. With this aim in mind, different groups of animals were injected with the Botulinum neurotoxin A (BoNT/A) (Supplemental material) before the beginning of training. BoNT/A is able to induce a long-lasting inhibition of neurotransmitter release by cleaving SNAP-25 (synaptosomal-associated protein of $25 \mathrm{kDa}$ ), an essential component of the vesicle fusion machinery (Schiavo et al. 2000; Rossetto et al. 2006; Antonucci et al. 2008). Figure 2 shows the expression levels of the cleaved SNAP-25 in the striatum 7 and $46 \mathrm{~d}$ after BoNT/A administrations. The cleaved protein was expressed in the striatum at both time points, confirming, in our conditions, the long lasting effects of the toxin (Schiavo et al. 2000; Rossetto et al. 2006; Antonucci et al. 2008). The analysis of the extension of the immunoreactivity, demonstrated that the mean values of the major axis (dorsoventral) and the minor axis length (mediolateral) were $1.1 \pm 0.07$ and $0.9 \pm 0.13 \mathrm{~mm}$, respectively. We estimated a spread of about $1.8 \mathrm{~mm}$ in the rostrocaudal direction, thus demonstrating that the effect was limited to the target area.

Consistent with previous findings in the literature (Yin and Knowlton 2004) pretraining DMS (Supplemental Fig. S2A) or DLS (Supplemental Fig. S2C) injection of BoNT/A had no effect or a negligible one on the percentage of correct trials during training (Days $\left[F_{(1,25)}=54.07 ; P<0.0001\right]$ and Treatment $\left[F_{(1,33)}=\right.$ 3.92; $P=0.056]$, respectively, for DMS and DLS). Although DMS deactivation slightly affected the percentage of animals using RS, we could not find any significant difference between the sham $(82 \%)$ and the DMS group (68\%) in the percentage of animals using the RS ( $\chi^{2}=1.01, P=$ n.s.) (Supplemental Fig. S2B). This is consistent with previous findings showing that DMS lesions do not affect the use of RS (Yin and Knowlton 2004). Both DMS (BoNT/A-injected) and sham (vehicle-injected) animals showed conditioned taste aversion to chocolate (Supplemental Fig. S1A,B). However, DMS mice generalized food aversion between the waiting cage and the maze more efficiently. RS from the familiar arm was not affected in reward-devalued DMS animals (Day $\left[F_{(1,33)}=6.17 ; P=0.02\right]$; Day $\times$ Outcome $\left[F_{(1,33)}=\right.$ 5.73; $P<0.02]$ ) (Fig. 3A). However, in contrast to devalued sham animals $\left(\chi^{2}=5.33 ; P=0.02\right)$ reward-devalued DMS animals maintained their turning response when released from the novel starting box ( $\chi^{2}=0.12 ; P=$ n.s.) (Fig. 3B). These findings demonstrate that intact synaptic transmission within the DMS is necessary to maintain the RS sensitive to the outcome value change from the novel arm. As already mentioned, the response observed after $26 \mathrm{~d}$ of training in sham reward-devalued animals when released from the novel arm suggests an interaction between motivational and spatial information. As a consequence, the preservation of the RS in devalued DMS mice allows one to speculate that this might be the site where spatial and outcome-related information are linked to the action, making it flexible according to motivational and contextual demands.

The analysis of the effects of DLS deactivation in a group of mice submitted to the $61 \mathrm{~d}$ of training shows, consistent with some previous findings (Botreau and Gisquet-Verrier 2010), but not others (Packard and McGaugh 1996; Packard 1999), no impairment in the use of RS in DLS (BoNT/A-injected) animals when compared with sham (vehicle-injected) controls $\left(\chi^{2}=\right.$ $0.03, P=$ n.s.) (Supplemental Fig. S2D). The mice that used RS in the two experimental groups were then submitted to the devaluation procedure. Devaluated DLS mice, as the sham group, developed conditioned taste aversion (Supplemental Fig. S1C,D). Nevertheless, on the last day of devaluation, the percentage of correct trials did not differ between the two groups when released from the start box used during training (Days $\left[F_{(1,20)}=12.58\right.$; $P=0.002]$ ) (Fig. 3C). Finally, devalued DLS mice, in contrast to

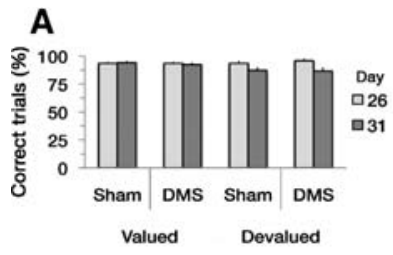

C

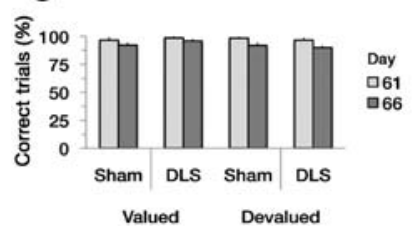

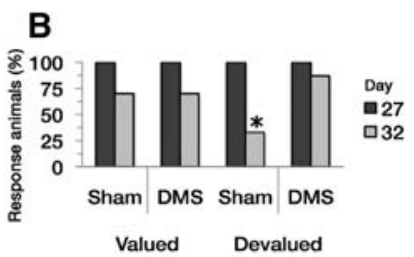

D

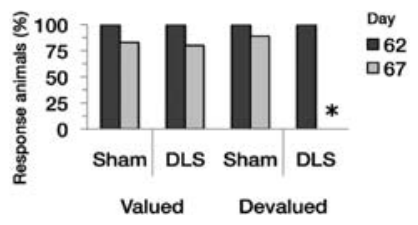

Figure 3. Dorsomedial (DMS) and dorsolateral (DLS) striatal deactivation effects on the use of response strategy after devaluation. (A) Percentage of correct trials in sham and DMS BoNT/A-injected groups, valued or devalued, on the last day of training (day 26) or on the last day of devaluation (day 31). (B) Percentage sham or DMS mice using a response strategy on probe test 1 (day 27), maintaining their response on probe test 2 (day 32 ) in the valued and devalued group. (C) Percentage of correct trials in sham and DLS BoNT/A-injected groups, valued or devalued, on the last day of overtraining (day 61) or on the last day of devaluation (day 66). (D) Percentage sham or DLS mice using a response strategy after overtraining on probe test 1 (day 62), maintaining their response on probe test 2 (day 67) in the valued and devalued group. $\left({ }^{*}\right) \quad P<0.05$ Tukey Honestly Significant Difference (HSD) post-hoc analysis to compare within group before and after devaluation-day 27 vs. day 32, or day 62 vs. day 67 . 
sham-devalued animals, shifted their response when starting from the novel arm $\left(\chi^{2}=4.00, P=0.045\right)$ (Fig. 3D). This result indicates that inactivation of DLS prevented the development of RS insensitivity to the interaction between the spatial change and reward devaluation promoted by overtraining. According to the current literature, this region plays a crucial role in S-R learning (Yin et al. 2005b, 2006). Therefore, one should expect impairment in the use of RS independently of the releasing arm or the devaluation procedure. The lack of effects on RS, observed in this study after $61 \mathrm{~d}$ of training, as well as after devaluation when mice were released from the familiar arm, suggests that an intact DLS might be required when novel stimulus-response learning is being formed, or when it is generalized to novel situations (e.g., a novel arm); with overtraining, however, its function might be bypassed by the activation of other brain regions (e.g., cerebellum) (Lancioni et al. 2010). The fact that DLS neural deactivation might not affect the acquisition of S-R association per se if the animals have more time to learn implies that this brain region might have a permissive, but not a indispensable role in habit formation under overtraining conditions.

In conclusion, in this study, by using a reward devaluation procedure we showed that animals using the RS, after $26 \mathrm{~d}$ of training changed their response when tested again after devaluation of the reward, indicating that they were not relying on S-R learning. Nevertheless, if the animals were overtrained for $61 \mathrm{~d}$, RS became completely independent on the hedonic value of the reward. This suggests that RS acquired in the dual-solution plus-maze task can rely on both A-O and S-R association, depending on the amount of training the animals receive. These findings challenge two general assumptions about the response strategy in the cross maze: (1) that the RS is always a form of S-R, (2) that the kind of learning mechanism that the animal is using from the training arm can be inferred by probing the animal from the novel arm. Finally, we showed that neural deactivation of the DMS and the DLS with BonT/A favored and abolished, respectively, the effects of practice on the sensitivity of the RS to outcome devaluation. Altogether, these results have relevant implications for the understanding not only of the neural basis of habit learning, but also of the learning mechanisms underlying different overt behaviors in this widely used maze task.

\section{Acknowledgments}

We thank S. Cabib, B.W. Balleine, and A. Pert for the useful discussion on the data presented in this study and for critically reviewing the manuscript, and Dr. G. Diez Roux for a critical revision of the text. We thank Dr. O. Rossetto and Prof. C. Montecucco for providing BoNT/A and the antibody against the cleaved form of SNAP-25. We also thank Elisa De Sanctis, Ilaria Palladino, Vladimiro Batocchi, and Angelo Grasso for their technical support. The present study has been supported by P.R.I.N. grants from M.I.U.R. (to A.O. and A. Mele) and grants DCMC and $\mathrm{SaC}$ from A.S.I. (to A.O. and A. Mele).

\section{References}

Adams CD. 1982. Variations in the sensitivity of instrumental responding to reinforcer devaluation. Q J Exp Psychol 34B: 77-98.

Adams CD, Dickinson A. 1981. Instrumental responding following reinforcer devaluation. Q J Exp Psychol 33B: 109-122.

Antonucci F, Rossi C, Gianfranceschi L, Rossetto O, Caleo M. 2008. Longdistance retrograde effects of botulinum neurotoxin A. J Neurosci 28: 3689-3696.

Balleine BW. 1992. The role of incentive learning in instrumental performance following shifts in primary motivation. J Exp Psychol: Anim Behav Proc 18: 236-250.
Botreau F, Gisquet-Verrier P. 2010. Re-thinking the role of the dorsal striatum in egocentric/response strategy. Front Behav Neurosci 4: 7. doi: 10.3389/neuro.08.007.2010.

Brightwell JJ, Smith CA, Neve RL, Colombo PJ. 2008. Transfection of mutant CREB in the striatum, but not the hippocampus, impairs long-term memory for response learning. Neurobiol Learn Mem 89: $27-35$.

Colombo PJ, Brightwell JJ, Countryman RA. 2003. Cognitive strategy-specific increases in phosphorylated cAMP response element-binding protein and c-Fos in the hippocampus and dorsal striatum. J Neurosci 23: 3547-3554.

Compton DM. 2004. Behavior strategy learning in rat: Effects of lesions of the dorsal striatum or dorsal hippocampus. Behav Processes 67: $335-342$.

De Leonibus E, Oliverio A, Mele A. 2005. A study on the role of the dorsal striatum and the nucleus accumbens in allocentric and egocentric spatial memory consolidation. Learn Mem 12: 491-503.

Ferragud A, Haro A, Sylvain A, Velázquez-Sánchez C, Hernández-Rabaza V, Canales JJ. 2010. Enhanced habit-based learning and decreased neurogenesis in the adult hippocampus in a murine model of chronic social stress. Behav Brain Res 210: $134-139$.

Franklin KBJ, Paxinos G. 1997. The mouse brain in stereotaxic coordinates. Elsevier Academic Press, San Diego, CA.

Kheirbek MA, Britt JP, Beeler JA, Ishikawa Y, McGehee DS, Zhuang X. 2009. Adenylyl cyclase type 5 contributes to corticostriatal plasticity and striatum-dependent learning. J Neurosci 29: 12115-12124.

Kosaki Y, Dickinson A. 2010. Choice and contingency in the development of behavioral autonomy during instrumental conditioning. J Exp Psychol Anim Behav Process 36: 334-342.

Lancioni A, Pizzo M, Fontanella B, Ferrentino R, Napolitano LM, De Leonibus E, Meroni G. 2010. Lack of Mid1, the mouse ortholog of the Opitz syndrome gene, causes abnormal development of the anterior cerebellar vermis. J Neurosci 30: 2880-2887.

Middei S, Geracitano R, Caprioli A, Mercuri N, Ammassari-Teule M. 2004a. Preserved fronto-striatal plasticity and enhanced procedural learning in a transgenic mouse model of Alzheimer's disease overexpressing mutant hAPPswe. Learn Mem 11: 447-452.

Middei S, Restivo L, Sgobio C, Passino E, Ammassari-Teule M. 2004b. Reversible inactivation of hippocampus and dorsolateral striatum in C57BL/ 6 and DBA/ 2 inbred mice failed to show interaction between memory systems in these genotypes. Behav Brain Res 154: 527-534.

Moustgaard A, Hau J. 2009. Induction of habits in rats by a forced-choice procedure in T-maze and the effect of pre-test free exploration. Behav Processes 82: 104-107.

Packard MG. 1999. Glutamate infused posttraining into the hippocampus or caudate-putamen differentially strengthens place and response learning. Proc Natl Acad Sci 96: 12881-12886.

Packard MG, McGaugh JL. 1996. Inactivation of hippocampus or caudate nucleus with lidocaine differentially affects expression of place and response learning. Neurobiol Learn Mem 65: 65-72.

Passino E, Middei S, Restivo L, Bertaina-Anglade V, Ammassari-Teule M. 2002. Genetic approach to variability of memory systems: Analysis of place vs. response learning and fos-related expression in hippocampal and striatal areas of C57BL/6 and DBA/2 mice. Hippocampus 12: 63-75.

Rossetto O, Morbiato L, Caccin P, Rigoni M, Montecucco C. 2006. Presynaptic enzymatic neurotoxins. J Neurochem 97: 1534-1545.

Schiavo G, Matteoli M, Montecucco C. 2000. Neurotoxins affecting neuroexocytosis. Physiol Rev 80: 717-766.

Tolman EC, Ritchie BF, Kalish D. 1946. Studies in spatial learning II. Place versus response learning. J Exp Psychol 36: 221-229.

Vanderschuren LJ, Everitt BJ. 2004. Drug seeking becomes compulsive after prolonged cocaine self-administration. Science 305: 1017-1019.

Yin HH, Knowlton BJ. 2004. Contributions of striatal subregions to place and response learning. Learn Mem 11: 459-463.

Yin HH, Knowlton BJ, Balleine BW. 2004. Lesions of dorsolateral striatum preserve outcome expectancy but disrupt habit formation in instrumental learning. Eur J Neurosci 19: 181-189.

Yin HH, Knowlton BJ, Balleine BW. 2005a. Blockade of NMDA receptors in the dorsomedial striatum prevents action-outcome learning in instrumental conditioning. Eur J Neurosci 22: 505-512.

Yin HH, Ostlund SB, Knowlton BJ, Balleine BW. 2005b. The role of the dorsomedial striatum in instrumental conditioning. Eur J Neurosci 22: $513-523$.

Yin HH, Knowlton BJ, Balleine BW. 2006. Inactivation of dorsolateral striatum enhances sensitivity to changes in the action-outcome contingency in instrumental conditioning. Behav Brain Res 166: $189-196$.

Received November 8, 2010; accepted in revised form February 2, 2011. 


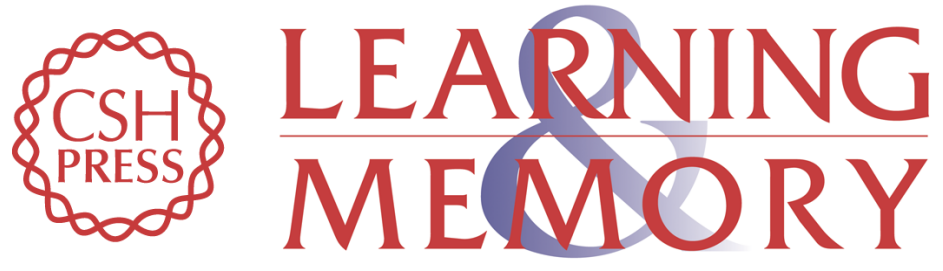

\section{Cognitive and neural determinants of response strategy in the dual-solution plus-maze task}

Elvira De Leonibus, Vivian J. A. Costantini, Antonio Massaro, et al.

Learn. Mem. 2011, 18:

Access the most recent version at doi:10.1101/lm.2074311 Supplemental http://learnmem.cshlp.org/content/suppl/2011/03/24/18.4.241.DC1
Material

References This article cites 28 articles, 9 of which can be accessed free at: http://learnmem.cshlp.org/content/18/4/241.full.html\#ref-list-1

License

Email Alerting Receive free email alerts when new articles cite this article - sign up in the box at the Service top right corner of the article or click here. 